\title{
Transverse profile tomography of a high current proton beam with a multi-wire scanner
}

\author{
Q. Z. Xing, ${ }^{1,2,3}$ L. Du, ${ }^{1,2,3,}$ X. L. Guan,,${ }^{1,2,3}$ C. X. Tang, ${ }^{1,2,3}$ \\ M. W. Wang, ${ }^{4}$ X. W. Wang, ${ }^{1,2,3}$ and S. X. Zheng ${ }^{1,2,3, *}$ \\ ${ }^{1}$ Key Laboratory of Particle and Radiation Imaging, Tsinghua University, \\ Ministry of Education, Beijing 100084, China \\ ${ }^{2}$ Laboratory for Advanced Radiation Sources and Application, \\ Tsinghua University, Beijing 100084, China \\ ${ }^{3}$ Department of Engineering Physics, Tsinghua University, Beijing 100084, China \\ ${ }^{4}$ State Key Laboratory of Intense Pulsed Radiation Simulation and Effect, \\ Northwest Institute of Nuclear Technology, Xi'an 710024, China
}

(Received 2 March 2018; published 20 July 2018)

\begin{abstract}
This paper describes the measurement of the transverse, two-dimensional profile of the high current proton beam in the linac at the Tsinghua University, by tomography mapping using a rotatable multi-wire scanner. The experiment is conducted at a beam energy of $3 \mathrm{MeV}$, a peak current of up to $32 \mathrm{~mA}$, a repetition frequency of $20 \mathrm{~Hz}$, and a beam pulse width of $50 \mu \mathrm{s}$. The transverse density distribution is reconstructed utilizing the algebraic reconstruction technique (ART), by using the beam profiles measured at different rotation angles. The beam profile, measured at a fixed rotation angle, is obtained by 19 parallel carbon wires mounted on one board of the wire scanner monitor (WSM) moving along a straight line with a step increment of $0.1 \mathrm{~mm}$. The results gathered from the computer tomography (CT) method could be verified by an independent profile measurement downstream of a multiple-slit aperture. This paper presents first measurement results of the high current proton beam during normal operation of the linac at the Tsinghua University. A dynamic range of $10^{2}$ for the two-dimensional distribution was achieved.
\end{abstract}

DOI: 10.1103/PhysRevAccelBeams.21.072801

\section{INTRODUCTION}

Wire scanner monitors (WSMs) are widely adopted in accelerator facilities for a minimum-invasive measurement of the transverse beam profile, with applications that include the understanding of the underlying beam dynamics, the determination of the emittance, and the beam halo [1-4]. The principle of the measurement is to obtain the flux information of the secondary particles scattered off the wires, by scanning the wire through the beam. This flux intensity is proportional to the primary proton beam colliding with the wires $[5,6]$. A one-dimensional transverse profile, projected perpendicular to the axes of wire motion, as obtained from a conventional wire-scanner can provide sufficient information for a beam with a Gaussian distribution. Nevertheless, a two-dimensional profile

\footnotetext{
*Corresponding author. zhengsx@tsinghua.edu.cn

${ }^{\dagger}$ Present address: CEA, IRFU, F-91191 Gif-sur-Yvette, France.

Published by the American Physical Society under the terms of the Creative Commons Attribution 4.0 International license. Further distribution of this work must maintain attribution to the author(s) and the published article's title, journal citation, and DOI.
}

measurement is necessary to study beams with irregular distributions, in particular high current beams.

The computerized tomography $(\mathrm{CT})$ to render $2 \mathrm{D}$ or $3 \mathrm{D}$ objects based on multiple 1D images from different angles is well known in the medical and industrial communities, and offers an effective method to measure the twodimensional distribution of charged particle beams in an accelerator. The one-dimensional data of projected beam profiles from different angles can be used to render the twodimensional cross-sectional images by computerized image reconstruction, based on the Radon transform [7]. Figure 1 presents the one-dimensional and two-dimensional transverse profile measurement by scanning the wire through the beam. CT imaging has been successfully applied to the diagnostics of electron beams, including the measurement of the transverse phase space using one or two solenoids $[8,9]$, the three-dimensional bunch profile using one $\mathrm{rf}$ deflecting cavity [10], and the two-dimensional beam profile using one rotatable multi-wire scanner [11].

The tomography mapping technology can also be exploited for the measurement of the transverse twodimentional distribution of ion beams. A rotatable multi-wire scanner has been developed at the Tsinghua University to measure the high current proton beam in a linac by applying a tomography mapping method. The one-dimensional 


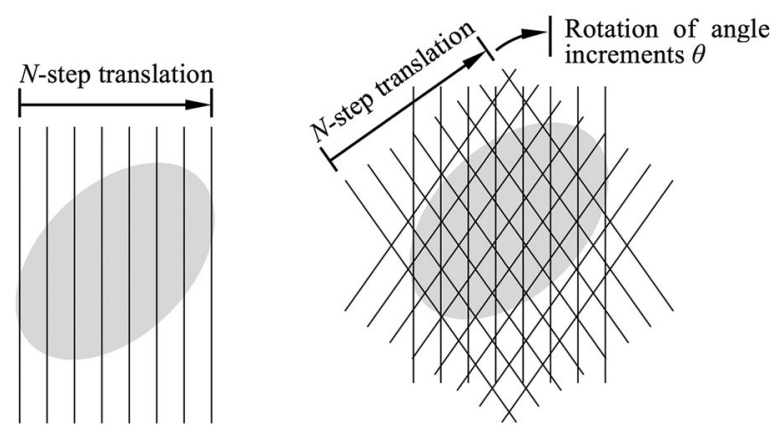

FIG. 1. One-dimensional (left) and two-dimensional (right) transverse profile measurement by scanning the wire through the beam.

projected beam profile is obtained by a parallel multi-wire arrangement, scanning the beam under a fixed angle. The transverse density distribution is reconstructed applying the algebraic reconstruction technique (ART) to the onedimensional beam profiles measured as projection under different rotation angles. The experiment is conducted at a beam energy of $3 \mathrm{MeV}$, a peak current of up to $32 \mathrm{~mA}$, a repetition frequency of $20 \mathrm{~Hz}$, and a beam pulse length of $50 \mu \mathrm{s}$.

This paper is organized in the following way. Section II describes the method of the transverse profile tomography of the high current proton beam with the multi-wire scanner. Section III presents the experimental setup. Section IV presents the experimental result and the verification of the method. Finally, the conclusions are summarized in Sec. V.

\section{THE MEASUREMENT METHOD}

The energy of the proton beam to be measured is $3 \mathrm{MeV}$. The material selected for the wires of $30 \mu \mathrm{m}$ diameter is carbon fiber, having a density of $1.98 \times 10^{3} \mathrm{~kg} / \mathrm{m}^{3}$. The penetration depth of $3 \mathrm{MeV}$ protons to carbon is $84.6 \mu \mathrm{m}$ [12], therefore the protons will pass through the carbon fiber wires, but will lose energy which results in the formation of internal secondary electrons. The process of secondary electron emission can be viewed as process composed out of two independent parts, namely the formation of secondaries and their subsequent escape [13]. As a result the impact of the protons on the carbon wires produces charges on the wires caused by the secondary electrons scattered off the wires. The external load impedance of the measurement system allows a current flow proportional to the scattered secondaries, which is also proportional to the primary proton beam colliding with the wires. As the first step of the tomography mapping, the current intensities on the 19 carbon wires mounted on a single board of the WSM are measured, and the corresponding data is acquired and saved while the board is moved along a straight line in steps of $0.1 \mathrm{~mm}$. Following, the carbon wire board is rotated by a fixed angle of $5^{\circ}$. This procedure is repeated until the total rotation angle reaches $160^{\circ}$. Finally the transverse two-dimensional distribution of the beam is reconstructed using the saved data of the current intensities for each wire board position.

\section{A. Secondary electron yield}

The yield of the secondary electrons is defined as the number of the secondary electrons scattered off the solid wire target per incident proton. The yield of the secondary electrons from a flat, homogeneous carbon surface induced by a proton beam of $3 \mathrm{MeV}$ under normal incident can be obtained from $Y \approx\left(P \cdot d_{s} / E^{*}\right) \mathrm{d} E / \mathrm{d} x$, where $P \approx 0.5$ is the probability of a secondary electron within the depth of $d_{s}$ below the surface to reach the surface and escape. In our case all secondaries are generated in a layer. $d_{s} \approx 1 \mathrm{~nm}$ is the depth below the surface where all the escaped secondary electrons are considered to be originated. $E^{*} \approx 25 \mathrm{eV}$ is the mean energy of the secondary electron, and $\mathrm{d} E / \mathrm{d} x$ is the mean energy loss of one proton per unit length. $\mathrm{d} E / \mathrm{d} x$ can be approximated as constant throughout the region within the depth of $d_{s}$ below the surface [13].

Meanwhile, for the carbon multi-wires, a cyclindrical model is used to estimate the yield of the secondary electrons, as shown in Fig. 2. The path of the primary protons is assumed to stay unchanged after they penetrate through the wires. Lets assume the current density of the incident proton beam to be $n$, and being constant over the wire. The number of the protons which impact the carbon wire within an angle between $\theta$ and $\theta+\mathrm{d} \theta$ is $n r \cos \theta \mathrm{d} \theta$, with $r$ being the radius of the wire. Then the travel length of the proton within a thin layer of depth $d_{s}(\approx 1 \mathrm{~nm})$ below the surface is $d_{s} / \cos \theta$.

The yield of the secondary electrons for the cylindrical carbon wire is composed of the yield from the incident surface and from the emergent surface:

$$
\begin{aligned}
Y & \approx \frac{P}{2 n r E^{*}} \int_{-\pi / 2}^{\pi / 2} n r \cos \theta \mathrm{d} \theta \frac{d_{s}}{\cos \theta} \frac{\mathrm{d} E}{\mathrm{~d} x} \\
& =\frac{P d_{s}}{2 E^{*}}\left[\left.\pi \frac{\mathrm{d} E}{\mathrm{~d} x}\right|_{E=3 \mathrm{MeV}}+\left.\int_{-\pi / 2}^{\pi / 2} \frac{\mathrm{d} E}{\mathrm{~d} x}\right|_{\theta} \mathrm{d} \theta\right]
\end{aligned}
$$

where the mean energy loss of one proton per unit length $\mathrm{d} E / \mathrm{d} x$ is considered to be constant on the incident surface, and a function of $\theta$ on the emergent surface. With $P=0.5$, $d_{s}=1 \mathrm{~nm}$, and $E^{*}=25 \mathrm{eV}$, the yield of the secondary

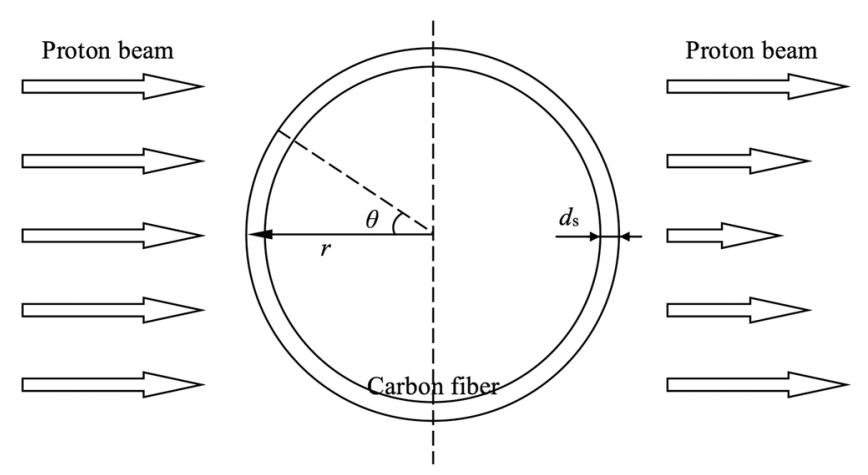

FIG. 2. Cylindrical model of the secondary electron emission. 
electrons is found to be $Y \approx 1.39$ for the cylindrical carbon wire with a diameter of $30 \mu \mathrm{m}$.

Taking that yield and results from beam dynamics, the current on the wire can be estimated. Assuming a peak current of the proton beam of $40 \mathrm{~mA}$, the maximum current produced on the wire is estimated to be $0.26 \mathrm{~mA}$, while moving the wire target through the center of the beam.

\section{B. Wire temperature}

The temperature of the wire will raise when the proton beam hits the wire. Significant thermionic emission and wire sublimation will emerge if the wire temperature is elevated to $1800 \mathrm{~K}$ and $2600 \mathrm{~K}$ respectively, which shall be avoided [14]. The wire temperature depends on the beam pulse width and repetition frequency, with other beam parameters unalterable. Therefore the beam pulse width and repetition frequency need to be carefully tuned according to the estimated wire temperature.

There are four cooling processes for the wire: radiation cooling, conduction cooling, thermionic cooling, and sublimation cooling [15]. The thermionic cooling and sublimation cooling can be neglected if the wire temperature stays below the threshold of the thermionic emission. Therefore, the thermal equilibrium equation of the wire in the proton beam can be described as Eq. (2):

$$
\begin{aligned}
P- & \epsilon \sigma\left(T^{4}-T_{0}^{4}\right) \cdot 2 \pi r \Delta x-\lambda \pi r^{2} \Delta x \frac{\mathrm{d}^{2} T}{\mathrm{~d} x^{2}} \\
& =C_{p} \rho \pi r^{2} \Delta x \frac{\mathrm{d} T}{\mathrm{~d} x}
\end{aligned}
$$

where $P$ is the power loss of a single macro pulse of the proton beam on the carbon fiber within the length of $\Delta x, \epsilon \approx 0.8$ is the black-body emissivity, $\sigma \approx 5.67 \times$ $10^{-8} \mathrm{~W} /\left(\mathrm{m}^{2} \cdot \mathrm{K}^{4}\right)$ is the Stefan-Boltzmann constant, $T$ is the wire temperature, $T_{0}=293 \mathrm{~K}$ is the environment temperature, $r$ is the wire radius, $\rho=1.98 \times 10^{3} \mathrm{~kg} / \mathrm{m}^{3}$ is the wire density, $\lambda$ is the thermal conductivity and $C_{p}$ is the heat capacity of the carbon [16] which can be obtained from:

$$
\begin{aligned}
\lambda= & 116.9-7.87 \times 10^{-2} T+2.062 \times 10^{-5} T^{2} \\
& -1.02 \times 10^{-10} T^{3}-4.153 \times 10^{-13} T^{4} \\
C_{p}= & 2025+7.872 \times 10^{-2} T-4.271 \times 10^{8} T^{-2} \\
& +1.322 \times 10^{11} T^{-3}-1.2 \times 10^{13} T^{-4}
\end{aligned}
$$

The maximum temperature of the wire reaches as the wire is moved through the center of the beam. The crosssection of protons and the wire target, i.e., the number of protons hitting the wire can be estimated by the simulation result of the transverse two-dimensional distribution of the proton beam at the position of the wires. With the total peak current of $40 \mathrm{~mA}$, the current through this wire is $0.184 \mathrm{~mA}$. The average power loss of one proton through

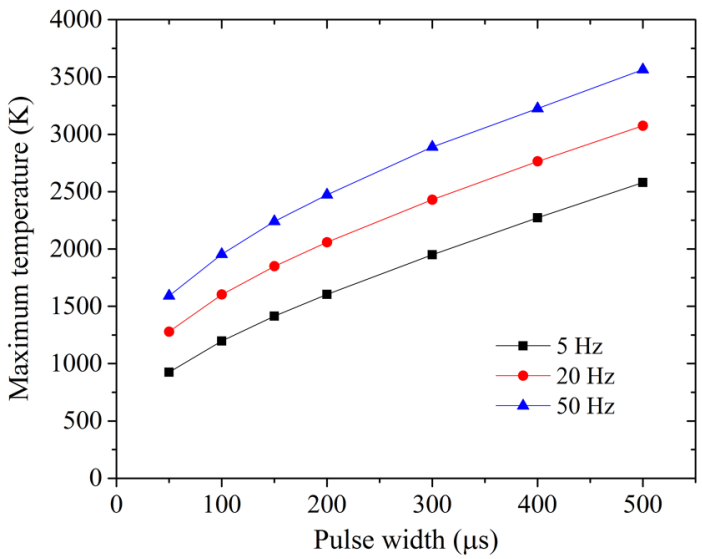

FIG. 3. Maximum temperature of the carbon wire with the pulse width and repetition frequency of the beam.

the wire is $0.53 \mathrm{MeV}$. Therefore, the total peak power produced by the proton beam on the wire through the beam center horizontally is about $97.5 \mathrm{~W}$. Figure 3 presents the maximum temperature of the carbon wire for different pulse widths and repetition frequencies of the beam, calculated numerically by Eq. (2). The maximum temperature increases with both, the beam pulse width and repetition frequency of the beam. To restrain thermionic emission and wire sublimation, the beam pulse width shall be no more than $50 \mu$ s for $50 \mathrm{~Hz}$ while $100 \mu$ s for $20 \mathrm{~Hz}$. With a beam pulse width of $50 \mu \mathrm{s}$ and a repetition frequency of $50 \mathrm{~Hz}$, the maximum temperature of the carbon wire varying with time is shown in Fig. 4. The maximum temperature reaches a steady state after several oscillation periods.

\section{Algebraic reconstruction technique}

The transverse density distribution is reconstructed applying the algebraic reconstruction technique (ART) using the one-dimensional projection profiles measured under different rotation angles. ART is one of the iterative

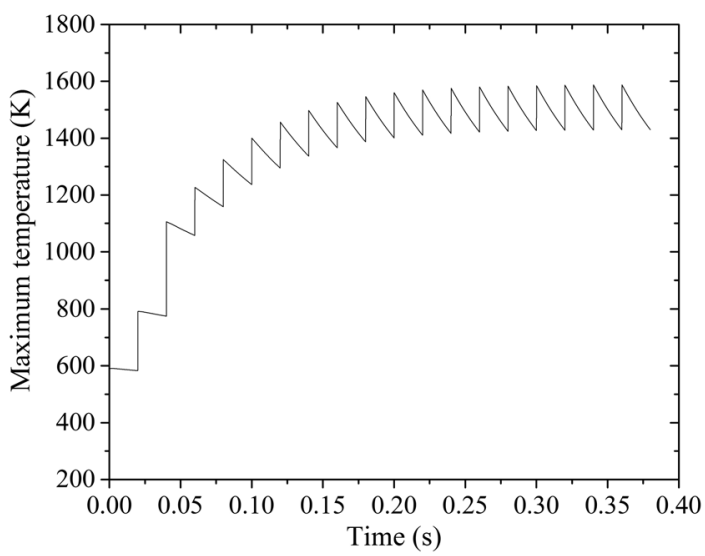

FIG. 4. Maximum temperature of the carbon wire with time (the beam pulse width is $50 \mu$ s and repetition frequency is $50 \mathrm{~Hz}$ ). 


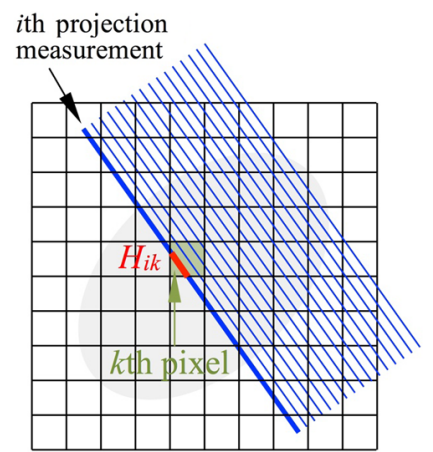

FIG. 5. Schematic diagram of the weight element $H_{i k}$ of the forward projection matrix $H . H_{i k}$ is calculated as the length of the intersection between the wire and the pixels.

reconstruction algorithms which is best suited for projections that are sparse, noisy or nonuniformly distributed [17]. Based on the algebraic method of ART [7,18,19], the currents measured on the wires can be illustrated as a set of algebraic equations

$$
H f=g
$$

where $f \in R^{N}$ denotes the $N$ discretized pixel values of the unknown transverse two-dimensional distribution of the beam, $g \in R^{M}$ are the $M$ values of the measured projection data (current), and $H \in R^{M \times N}$ is the forward projection matrix mapping the unknown distribution function $f$ to the projection $g$ being measured. Equation (5) is linear and can be solved using ART by sequentially projecting a solution estimate onto the hyperplanes defined by each row of the linear system. The iteration process can be expressed mathematically by

$$
\begin{aligned}
f_{j}^{(n+1)} & =f_{j}^{(n)}+\alpha \frac{g_{i}-\sum_{k=1}^{N} H_{i k} f_{k}^{(n)}}{\sum_{k=1}^{N} H_{i k}^{2}} H_{i j}, \\
i & =1, \ldots, M
\end{aligned}
$$

where $f_{j}^{(n)}$ is the $n$th estimate of the $j$ th pixel values of the distribution, $g_{i}$ is the measured current on the $i$ th wire, $\alpha$ is the relaxation factor, and $H_{i k}$ is the $i k$ th element of the weight matrix $H$. The contribution of one pixel $k$ to the measurement $i$ can be obtained as the length of the intersection between the wire and the pixels, as shown in Fig. 5. Equation (6) represents the projection of $f_{j}^{(n)}$ on the hyperplane represented by the $(n+1)$ th equation to yield $f_{j}^{(n+1)}$.

\section{EXPERIMENTAL SETUP}

The wire scanner monitor system consists out of the multi-wires, the necessary mechanics to precisely move and rotate the multi-wire board, and the electronics system to acquire, process and store the current signals. The entire system is installed in the high energy beam transport (HEBT) line of the proton linac, which is part of the compact pulsed hadron source (CPHS) at the Tsinghua University, downstream the $3 \mathrm{MeV}$ accelerator [20].

\section{A. Multi-wire assembly and motion control}

Nineteen carbon wires, each having a diameter of $30 \mu \mathrm{m}$ are aligned and mounted on a board, as shown in Fig. 6(a). The pitch between the wires is $3 \mathrm{~mm}$. The multi-wires can be rotated around the center of the board controlled by a step motor and gear system, which are in vacuum, as shown in Figs. 6(b) and 6(c). Two limit switches are adopted to restrict the rotation range. Outside the vacuum chamber a second step motor is used to move the system linearly, with help of a threaded rod, a bearing and a set of bellows, as illustrated in Fig. 6(d). The rod in Fig. 6(c) connecting with the bellows can move up and down with the bellows. Also for this linear motion the range is restricted by two limit switches. The computer controlled movements of the multiwire board for a tomographical scan are initiated by setting the linear axes step increment $a$ (in $\mathrm{mm}$, dividable by 3 ). Now a rotation scan of angle increments $\theta$ is performed, followed by a linear motion for $3 / a-1$ steps along the rod in Fig. 6(b), with in step increments of $a / \cos \theta$. For each step the wire currents are measured and the data is stored.

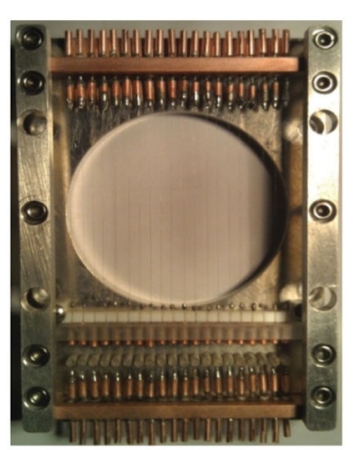

(a) Multi-wires on one board

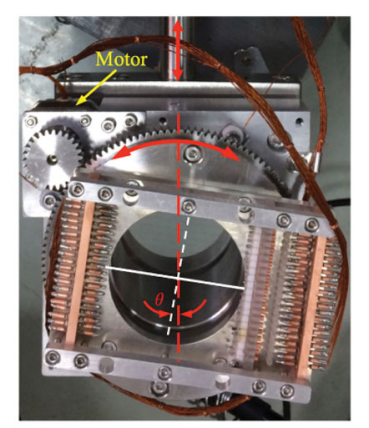

(c) Structure in vacuum

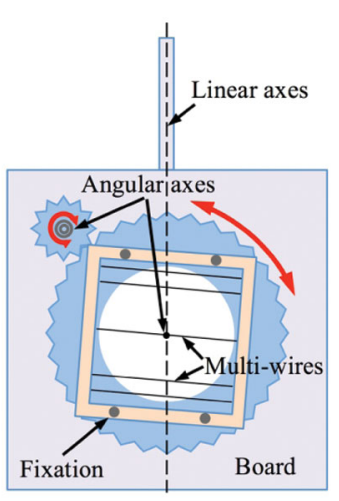

(b) Schematic graph for the motion

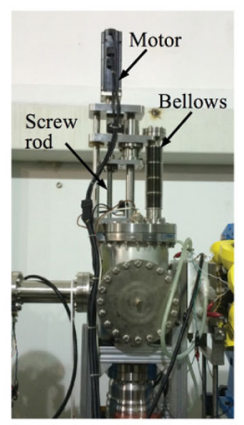

(d) Structure in air
FIG. 6. Mechanical design of the rotatable wire scanner monitor. 


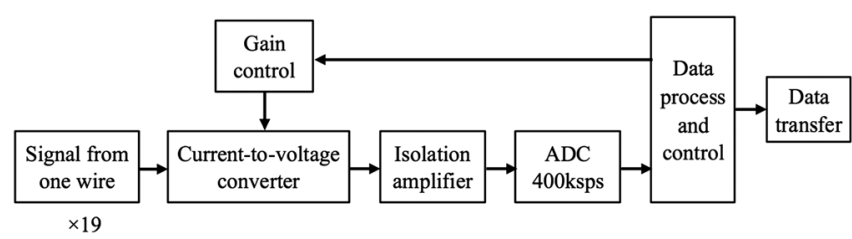

FIG. 7. Schematic design of the electronics system for the wire scanner monitor.

\section{B. Control and read-out electronics}

The wire-current read-out electronics needs to cover a current range of $5 \mathrm{nA}$ to $500 \mu \mathrm{A}$, has to indicate a broken wire and should digitize the signal at a sampling rate of $400 \mathrm{ksps}$. The schematic design of the electronics system for the wire scanner monitor is shown in Fig. 7. Noise minimization is critical for a precise measurement, therefore the signal lines are separated and well shielded from the motor control cables. This includes the shielding of the connectors as well.

Electronic cross talk between the individual wire channels due to secondary electron emission and capacitive coupling must be considered during the acquirement of the signals from the wires. One bias voltage of $-30 \mathrm{~V}$ is applied to the wires, which prevents the secondary electrons scattered off the wires from returning back to the wires. The capacitive coupling will lead to an overshoot at the beginning of the pulse signal of the impacted wire. Figure 8 presents three acquired signals from Wire 12, 13, and 14, at a sampling rate of $400 \mathrm{ksps}$. The cross-talk effect can be observed on the two signals from Wire 12 and 14. The reduction of the noise can be achieved by sampling the pulse signal beyond the overshooting region. To reduce this effect the effective signal is obtained by the average of the amplitudes of the sampled points from 25th to 31 st.

\section{Experimental configuration}

The measurement of the transverse two-dimentional profile of the high current proton beam by tomography

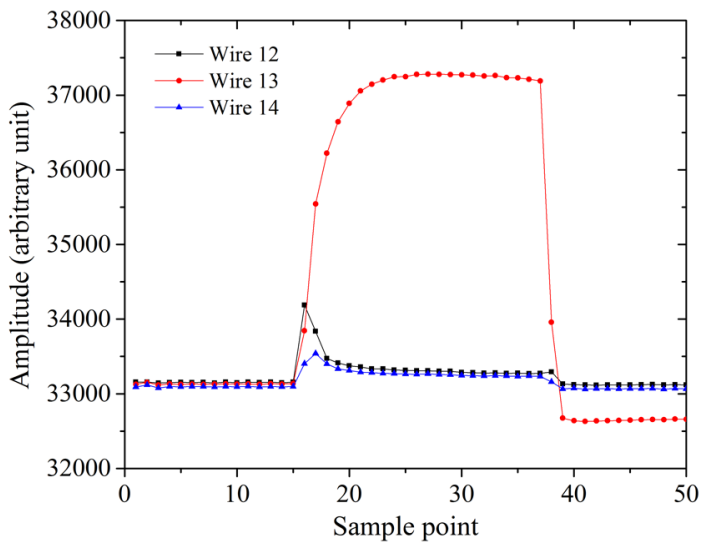

FIG. 8. Acquired signals from Wire 12, 13, and 14, at a sampling rate of $400 \mathrm{ksps}$. A bias voltage of $-30 \mathrm{~V}$ is applied to the wires.

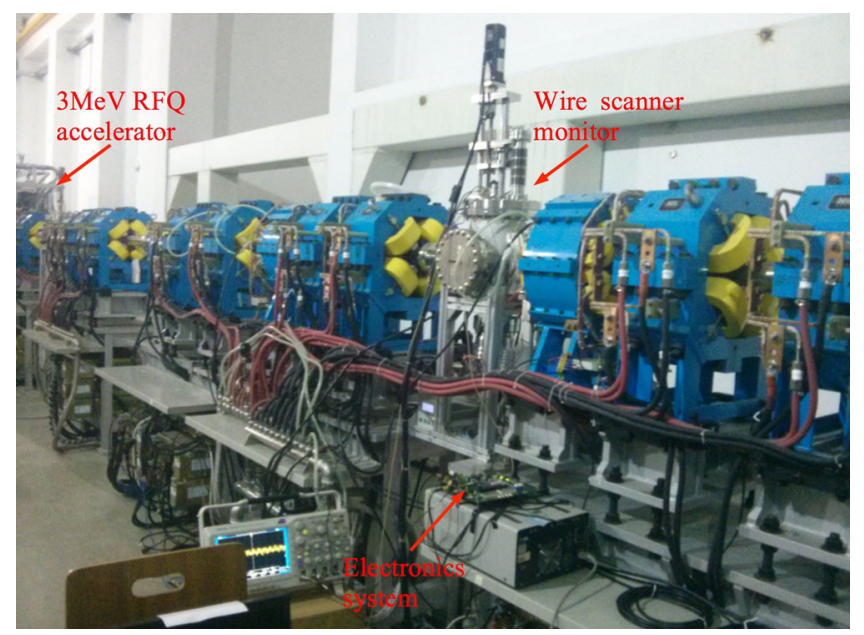

FIG. 9. Experimental setup of the wire scanner monitor.

mapping was performed in the HEBT line of the proton linac of the compact pulsed hadron source at the Tsinghua University. Main elements of the linac are the ECR Ion Source (IS), the low energy beam transport line (LEBT), the 4-vane RFQ accelerator, the HEBT line, and the rf power supply and distributor. A proton beam with an energy of $3 \mathrm{MeV}$, a peak current of up to $32 \mathrm{~mA}$, a repetition frequency of $20 \mathrm{~Hz}$, and a beam pulse duration of up to $500 \mu$ s can be provided. Figure 9 shows the installed wire scanner monitor in the HEBT line.

\section{MEASUREMENT RESULTS}

\section{A. Verification experiment}

To verify the method of the transverse profile tomography of the high current proton beam with the rotatable multi-wires, the first measurement was performed by shaping the beam using an especially designed multi-slit aperture, as shown in Fig. 10. There are totally seven slits on the aperture, with a slit width of $2 \mathrm{~mm}$, and a pitch of $4 \mathrm{~mm}$. The distance between the aperture and the multiwires is approximately $25 \mathrm{~cm}$.

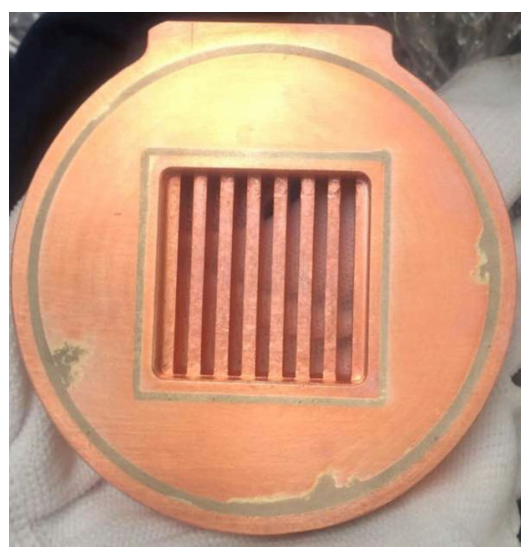

FIG. 10. Multi-slit aperture for the verification measurement. 


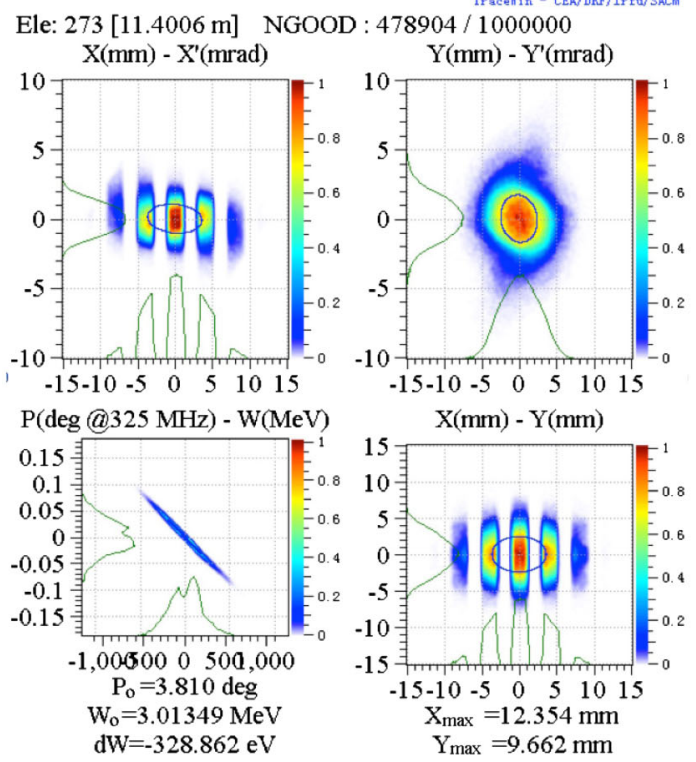

(a) At the position of the aperture

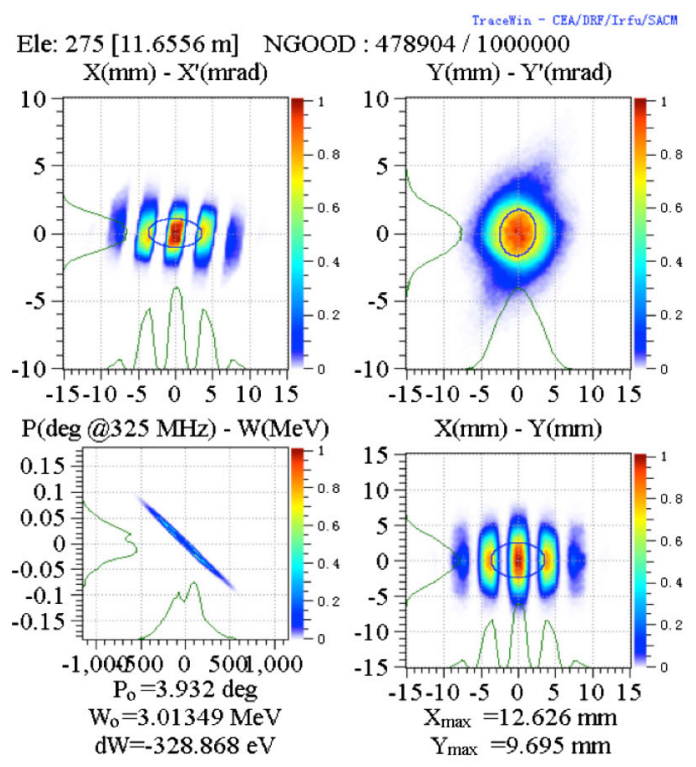

(b) At the position of the multi-wires

FIG. 11. Phase space and transverse profile of the proton beam at the positions of the aperture and multi-wires simulated by the TRACEWIN code.

The quadrupoles in the HEBT have been adjusted to produce a flat beam in the transverse plane at the position of the aperture. The phase space and transverse profile of the proton beam at the positions of the aperture and multi-wires have been investigated with help of the TRACEWIN beam dynamics simulation code [21], as presented in Fig. 11.

Figure 12 presents the reconstructed transverse profiles of the high-current proton beam by the computerized tomography imaging with/without the aperture upstream the rotatable multi-wires. The experiment is conducted at a repetition frequency of $10 \mathrm{~Hz}$, and a beam pulse duration of

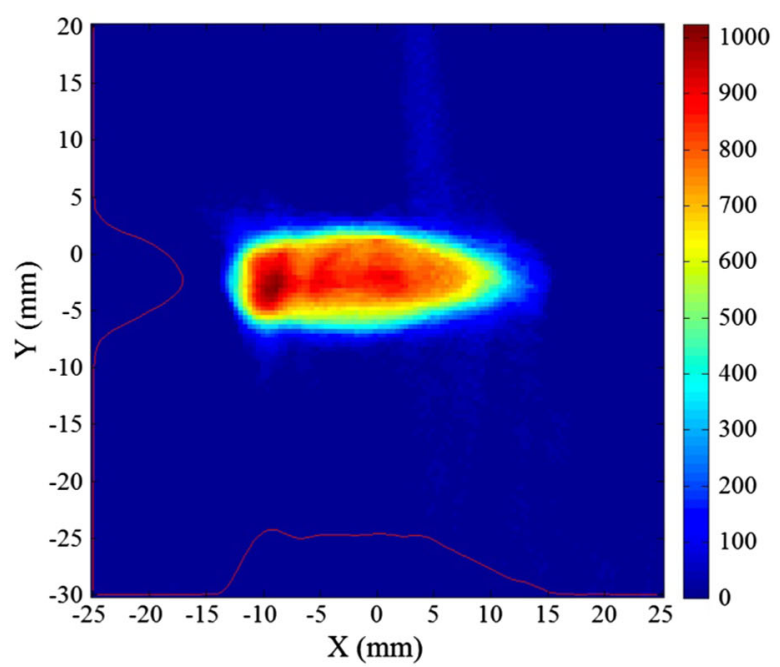

(a) Without the aperture

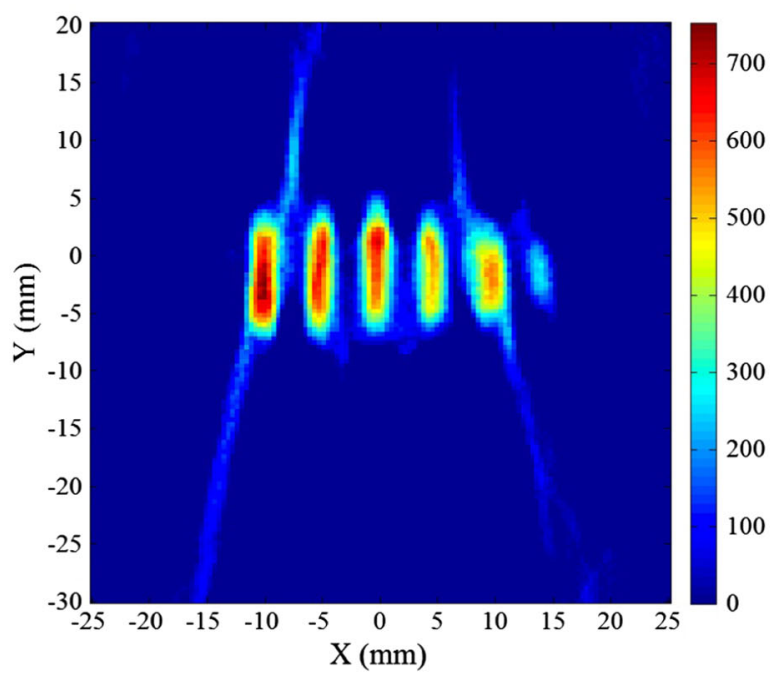

(b) With the aperture

FIG. 12. Reconstructed transverse profiles of the proton beam by the computerized tomography imaging with/without the aperture upstream the rotatable multi-wires.

$50 \mu \mathrm{s}$. The position step and angle step of the multi-wires for the computerized tomography is $0.1 \mathrm{~mm}$ and $5^{\circ}$ respectively. The total rotation angle is $160^{\circ}$. The computerized tomography result conforms to the predicted profile, with respect to the simulation result in Fig. 11.

\section{B. Measurement during normal beam operation}

The transverse profile of the proton beam of the CPHS linac is measured during the normal operation of the linac. The peak current measured by the ACCT reaches $32 \mathrm{~mA}$, as shown by the purple line in Fig. 13. These measurements are performed under the same beam and measurement conditions as the verification experiment, except that the repetition frequency is increased to $20 \mathrm{~Hz}$. The parameters of the multi-wires are the same with the verification experiment. The total measurement time is $25 \mathrm{~min}$. 


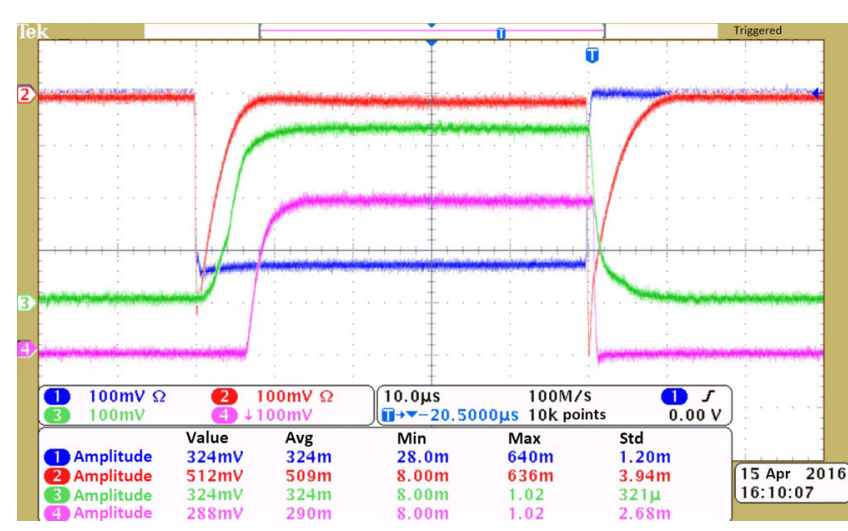

FIG. 13. Normal operation of CPHS linac. (blue line: input power fed into the RFQ; red line: reflected power of the RFQ; purple line: ACCT signal at the exit of the RFQ; green line: ACCT signal at the exit of the HEBT line.

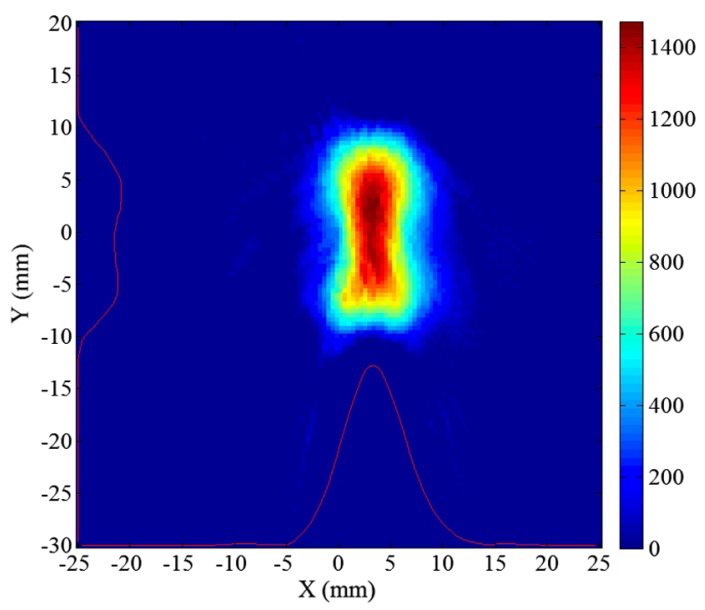

FIG. 14. Reconstructed transverse profiles of the proton beam by the computerized tomography imaging during the normal operation of the CPHS linac, with the beam energy of $3 \mathrm{MeV}$, repetition frequency of $20 \mathrm{~Hz}$, and beam pulse width of $50 \mu \mathrm{s}$.

The reconstructed transverse profiles of the proton beam by the computerized tomography imaging is shown in Fig. 14. A dynamic range of $10^{2}$ for the transverse twodimensional distribution could be achieved.

\section{CONCLUSIONS}

As one of the minimum-invasive beam profile measurement methods, the computerized tomography imaging with the rotatable multi-wire scanner provides an effective new approach to obtain the two-dimensional profile of the high current ion beams. Compared with the conventional wire scanner, the advantage of this approach lies in the fact that a two-dimensional image of the particle density can be reconstructed.

With the focus of the measurement on a high current proton beam, we established a model to calculate the secondary electron yield and the temperature of the carbon wires. To restrain thermionic emission and wire sublimation, the beam pulse width has been revealed to be no more than $50 \mu \mathrm{s}$ for a repetition frequency of $50 \mathrm{~Hz}$, while $100 \mu$ s are possible for $20 \mathrm{~Hz}$. A wire scanner monitor system has been manufactured successfully. The wirescanner monitor contains nineteen parallel carbon wires with the diameter of $30 \mu \mathrm{m}$ and a pitch of $3 \mathrm{~mm}$ between two adjacent wires.

The method of the transverse profile tomography of the high current proton beam with the rotatable multi-wires was verified by measuring the beam profile, shaped using an especially designed multi-slit aperture. The experiment was performed at a beam energy of $3 \mathrm{MeV}$, a repetition frequency of $10 \mathrm{~Hz}$, and a beam pulse width of $50 \mu \mathrm{s}$. The position step and angle step of the multi-wires for the computerized tomography was $0.1 \mathrm{~mm}$ and $5^{\circ}$ respectively. The total rotation angle was $160^{\circ}$. The computerized tomography result verified the predicted profile, with respect to the simulation result. It is worth stressing that the algebraic reconstruction technique has been verified to be an effective reconstruction algorithm for this approach.

During the normal operation of the CPHS linac at the Tsinghua University, the transverse profile of the proton beam in the HEBT line was measured. The first reconstructed transverse profiles of the proton beam with the peak current of $32 \mathrm{~mA}$ by the computerized tomography imaging was obtained successfully. With the repetition frequency of $20 \mathrm{~Hz}$, the total measurement time was $25 \mathrm{~min}$. The time can be further reduced to $17 \mathrm{~min}$ if the repetition frequency increases to $50 \mathrm{~Hz}$. A dynamic range of $10^{2}$ for the transverse two-dimensional distribution could be achieved.

\section{ACKNOWLEDGMENTS}

The authors are grateful to Liang Li for fruitful discussions on the Algebraic Reconstruction Technique and Xiaoshan Jiang for providing the electronics system. The authors also would like to thank Qingzhu Zhang for the operation of the proton linac. This work was supported by the National Natural Science Foundation of China (Major Research plan Grant No. 91126003).

[1] C. K. Allen, K. C. D. Chan, P. L. Colestock, K. R. Crandall, R. W. Garnett, J. D. Gilpatrick, W. Lysenko, J. Qiang, J. D. Schneider, M. E. Schulze, R. L. Sheffield, H. V. Smith, and T. P. Wangler, Beam-Halo Measurements in High-Current Proton Beams, Phys. Rev. Lett. 89, 214802 (2002).

[2] J. D. Gilpatrick, D. Barr, L. A. Day, D. M. Kerstiens, M. Stettler, R. Valdiviez, M. Gruchalla, J. F. O'Hara, and J. H. Kamperschroer, in Proceedings of the 19th Particle Accelerator Conference, Chicago, IL, 2001 (IEEE, Piscataway, NJ, 2001), p. 525. 
[3] K. Wittenburg, in Proceedings of HB2006 (Tsukuba, Japan, 2006), p. 54.

[4] A. Miura, H. Sako, H. Akikawa, and M. Ikegami, in Proceedings of the International Particle Accelerator Conference, Kyoto, Japan (ICR, Kyoto, 2010), p. 1008.

[5] P. Elmfors, A. Fasso, M. Huhtinen, M. Lindroos, J. Olsfors, and U. Raich, Wire scanners in low energy accelerators, Nucl. Instrum. Methods Phys. Res., Sect. A 396, 13 (1997).

[6] S. G. Arutunian, M. R. Mailian, and K. Wittenburg, Vibrating wires for beam diagnostics, Nucl. Instrum. Methods Phys. Res., Sect. A 572, 1022 (2007).

[7] A. C. Kak and M. Slaney, Principles of Computerized Tomographic Imaging (IEEE Press, New York, 1988).

[8] C. B. McKee, P. G. O'Shea, and J. M. J. Madey, Phase space tomography of relativistic electron beams, Nucl. Instrum. Methods Phys. Res., Sect. A 358, 264 (1995).

[9] D. Xiang, Y. C. Du, L. X. Yan, R. K. Li, W. H. Huang, C. X. Tang, and Y.Z. Lin, Transverse phase space tomography using a solenoid applied to a thermal emittance measurement, Phys. Rev. ST Accel. Beams 12, 022801 (2009).

[10] J. R. Shi, Y. C. Du, W. H. Huang, C. X. Tang, H. B. Chen, and L.X. Yan, Reconstruction of the three-dimensional bunch profile by tomography technique with RF deflecting cavity, Nucl. Instrum. Methods Phys. Res., Sect. A 752, 36 (2014).

[11] N. J. Yu, C. X. Tang, S. X. Zheng, Q. F. Li, and K. Gong, 2-D beam profile measurement based on CT algorithm with rotating multi-wire target, High Energy Phys. Nucl. Phys.-Chin. Ed. 29, 408 (2005).
[12] "http://www.srim.org,".

[13] E. J. Sternglass, Theory of secondary electron emission by high-speed ions, Phys. Rev. 108, 1 (1957).

[14] T. Yang, S. N. Fu, T. G. Xu, Z. H. Xu, M. Meng, R. Y. Qiu, J. M. Tian, L. Zeng, P. Li, F. Li, and B. Wang, Thermal analysis for wire scanners in the CSNS Linac, Nucl. Instrum. Methods Phys. Res., Sect. A 760, 10 (2014).

[15] M. Sapinski, Model of carbon wire heating in accelerator beam, CERN Technical Report No. CERN-AB-2008-030BI, 2008.

[16] A. T. Dinsdale, SGTE data for pure elements, CALPHAD: Comput. Coupling Phase Diagrams Thermochem. 15, 317 (1991).

[17] F. Q. Yang, D. H. Zhang, K. D. Huang, Z. Z. Gao, and Y. F. Yang, Incomplete projection reconstruction of computed tomography based on the modified discrete algebraic reconstruction technique, Meas. Sci. Technol. 29, 025405 (2018).

[18] X. Intes, V. Ntziachristos, J. P. Culver, A. Yodh, and B. Chance, Projection access order in algebraic reconstruction technique for diffuse optical tomography, Phys. Med. Biol. 47, N1 (2002).

[19] L. Zhang, X. H. Duan, Y. X. Xing, Z. Q. Chen, and J. P. Cheng, in Proceedings of SPIE 2008, Vol. 6913, p. $69132 \mathrm{~W}$.

[20] X. W. Wang, Q. Z. Xing, C.-K. Loong, X. L. Guan, and T. B. Du, Delivery of 3-MeV proton and neutron beams at CPHS: A status report on accelerator and neutron activities at Tsinghua University, Phys. Procedia 60, 186 (2014).

[21] D. Uriot, Tracewin documentation, CEA Saclay Technical Report, 2014. 